\title{
Laboratory testing in general practice: a patient safety blind spot
}

\author{
Nancy C Elder
}

Correspondence to Dr Nancy C Elder, Department of Family and Community Medicine, University of Cincinnati College of Medicine, P.0. Box 670582, Cincinnati, OH 45267-0582, USA; Nancy.elder@uc.edu

Accepted 28 July 2015 Published Online First 18 August 2015

\section{SLinked}

- http://dx.doi.org/10.1136/ bmjas-2014-003690

- http://dx.doi.org/10.1136/ bmjqs-2014-003712

- http://dx.doi.org/10.1136/ bmjqs-2015-004645

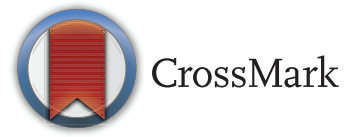

To cite: Elder NC. BMJ Qual Saf 2015;24:667-670.
Physicians order a lot of tests in primary care. We use tests to diagnose patients' complaints, monitor chronic diseases, check medication levels and screen for health risks and early disease. Sometimes, even in the office setting, the result for a test can be a matter of life or death. But more often, whether the results get reviewed by the physician and to the patient today, tomorrow or even next week, may not matter much to the physician or to the patient. Unfortunately, this laisser-faire attitude about test results has led to clinical offices where missing and lost test results, no patient notifications and lack of follow-up constitute the norm.

In the USA, studies in the late 1990s began delineating problems in the primary care testing process. ${ }^{1-3}$ In the following decade and a half, work by several teams of researchers brought more clarity about testing process errors, the harms they may cause and the role of cascading errors, mitigation and recovery. ${ }^{4-15}$ Litchfield et al ${ }^{16}{ }^{17}$ set out to understand the status of the testing process in British general practice (GP) offices. Using focus groups of patients and GP staff, the authors previously published the separate views of the two groups about how test results are communicated to patients by the offices. In their current studies published in BMJ Quality \& Safety journal, ${ }^{18} 19$ they present suggestions offered by GP staff and patients to improve the testing process and describe the findings of a survey of GP office staff (lead receptionists, practice managers) in order to expand our understanding of how test results are communicated with patients in GP offices.

The new studies have some significant limitations, including an inability to populate the focus groups with the purposeful sample desired, no attempt to reach data saturation, and a convenience sample for the survey. Nonetheless, the studies' findings pass what one might call a 'sniff test' - they are believable and clinically convincing. ${ }^{20}$ The detailed descriptions and rich participant quotes add credibility-a key criteria in evaluating qualitative research. ${ }^{21} 22$ The findings are also highly consistent with results from previous studies. For example, in our own 2006 study of testing process problems as seen by physicians and their staff, a participant noted that, "if a patient doesn't call and say, I haven't heard about my test results, we really don't know that they're not back." 6 Similarly, quoting a GP in a study by Litchfield, ${ }^{19}$ "If the patient hasn't called for the result we may never know that they didn't get the result."

The fact that the problems described in these articles continue to plague primary care practices and their patients requires an understanding of some of the key factors in the sociotechnical work system of the testing process in primary care: physicians and staff and their work culture; testing process technology; and patients and families and their culture.

An important advance of patient safety over the last two decades has been an understanding of team dynamics in achieving safe, quality patient outcomes. While originating in fast moving and time limited areas like emergency departments, intensive care units and operating rooms, team based care is a key component in the Patient Centered Medical Home movement in the USA and elsewhere. ${ }^{23}$ But with regards to the primary care testing process, Litchfield ${ }^{18} 19$ reveals a linear set of tasks performed by individuals often unaware of the steps that occur before or later in the process, a situation noted in other studies as well. $^{7}{ }^{8}{ }^{24}$ And the testing process doesn't occur over hours, as in the hospital, but over days and even weeks. Everyday in primary care practice, information chaos, including information on 
test orders and results, is the norm. As described by Beasley et $a l,{ }^{25}$ information chaos consists of information overload, underload, scatter, conflict and erroneous information and leads to impaired situational awareness and increased mental workload. Missing laboratory results are a common part of the underload; they were reported in $6.1 \%$ of all office visits in a Colorado study. ${ }^{26}$ Frequent minimally abnormal findings are part of the overload; fewer than half of abnormal glucose values were followed up in primary care study of laboratory results in non-diabetic patients. ${ }^{27}$ When overwhelmed by such information chaos, without a functioning team to help put some order to the chaos, individual physicians have to make decisions about where to focus their limited time and energy. As these studies of the testing process reveal, GPs often depend on untrained and ill-prepared staff to relay clinical information to patients and on patients to tell them when results are missing. ${ }^{18} 19$ Although missing or delayed test results have traditionally been seen by researchers as medical errors, they are not necessarily perceived that way by practicing physicians. Because these events rarely lead to significant patient harm, occur commonly and are part of a complex system, practicing physicians are much less likely to consider them medical errors at all, and thus tolerate the frequent problems in their practices. ${ }^{28}$

In the early 2000s, at least in the USA, primary care physicians hoped that electronic health records (EHR) would provide the answer to their ongoing frustrations with testing process problems. ${ }^{6}$ This obviously has not proven to be the case, or British GPs, most of whom have had electronic records longer than their American counterparts, would not be having the problems described by Litchfield et al. Yet technology does need to be part of the solution, especially for two areas described by Litchfield ${ }^{18}{ }^{19}$ as the lack of a 'failsafe' or tracking system (assuring that every test order results in a test result) and patient notification of test results.

The electronic transfer of orders and results has decreased lost and misplaced paper forms and misfiles. Too often, however, EHRs integrate these two processes without thoughtful functionalities for automated tracking between orders and results, as well as automated alerts for orders that don't match to a timely result. ${ }^{9}$ This leaves practices having to develop their own tracking systems, using valuable staff time and resources. Technology can also assist in the transmission of results to patients. While not all patients have access to or comfort with computers, a significant number do, and patient portals offer a way for physicians to communicate with many patients in a timely manner, especially for normal results. Patients are generally most pleased with direct physician communication of test results, but they also know that is not realistic and express satisfaction with any of a number of options, including patient portals and other electronic methods. ${ }^{29-33}$ Not surprisingly, in no studies did patients express a preference for calling for their own results, the most common method used in the GP offices as reported by Litchfield. ${ }^{19}$ This is a serious and important issue for British GPs, and if this small sample is indicative of the majority of practices, then GP patients are at significant risk of not being notified of important results that can affect their health. While it is appropriate for patients to serve as double checkers, ("if you don't hear from us with your results in 5 days, please call us"), it is inappropriate for physicians and staff to offload their responsibility for timely and appropriate communication of test results onto their patients.

Online access by patients to their own records, including test results, is rolling out across Britain now, and the lack of such practices in the Litchfield et al studies is a weakness. Further studies including offices already using online portals for patients could help GPs best transition to a system that uses technology well and doesn't embrace more errors. Just as EHRs that don't have automatic tracking of test orders don't help GPs failsafe their testing process, neither does an online patient portal that doesn't automatically report whether patients have logged in and viewed their results.

The inclusion of patients in the studies of GP testing process is important to understand the entire process. Unfortunately, while these studies confirm the problems experienced by GP's patients in navigating the testing process, they don't tell us what patients are doing about these problems. Patient-centred care would have us revolve our care around the patient, not the physician. Empowering patients to know what tests are being recommended and how the results will inform care, with shared decision making about test ordering may make patients more likely to complete the test, and to better understand the results they receive. ${ }^{34}$ Whether results are relayed by a phone call, letter or via an online patient portal, patients want to know what the result means for them, and what actions, if any, they should take on the result. ${ }^{30}$ We need further research to delineate the best ways for GP offices to advance from (1) having results available in the office for patients who call, to (2) notifying every patient of every result, to (3) partnering with patients to make the results and the physician's interpretation and recommendations part of shared decision making around care. So why aren't patients clamouring for such a system? A small study of patients experiencing preventable problems in primary care found that patients' behavioural responses fell into four categories: avoidance (eg, stop going to the doctor), accommodation (eg, learn to deal with delays), anticipation (eg, attend to details) and advocacy (eg, try to make a change). ${ }^{35}$ Patients in these studies described several of these responses around 
the testing process, especially accommodation (making the appointment at the reception desk, but knowing it will be a 2 week wait) and anticipation (not accepting results from a receptionist unless it is a cholesterol). ${ }^{18}$ Improving the GP testing process also means helping our patients become self-advocates for their own patient safety by involving them in care and shared decision making.

Laboratory testing will remain a common and important part of primary care. Unfortunately, for British GPs, these studies demonstrate that advances in patient safety in the last 20 years seem to have bypassed the testing process in primary care. By shedding needed light on dysfunctional processes, there is hope that patients, physicians and staff, together with advances in technology, will find ways to ensure that laboratory testing becomes patient-centred, and that ordering, tracking, patient notification and follow-up processes are transformed into safe practices that enhance patient care.

Competing interests None declared.

Provenance and peer review Commissioned; internally peer reviewed.

\section{REFERENCES}

1 Boohaker EA, Ward RE, Uman JE, et al. Patient notification and follow-up of abnormal test results. A physician survey. Arch Intern Med 1996;156:327-31.

2 Mold JW, Cacy DS, Dalbir DK. Management of laboratory test results in family practice. An OKPRN study. Oklahoma Physicans Resource/Research Network. J Fam Pract 2000;49:709-15.

3 Nutting PA, Main DS, Fischer PM, Jr, et al. Toward optimal laboratory use. Problems in laboratory testing in primary care. JAMA 1996;275:635-9.

4 Casalino LP, Dunham D, Chin MH, et al. Frequency of failure to inform patients of clinically significant outpatient test results. Arch Intern Med 2009;169:1123-9.

5 Chen E, Eder M, Elder N, et al. Crossing the finish line: follow up of abnormal test results in a multi-site community health center. J Natl Med Assoc 2010;102:720-5.

6 Elder N, Graham D, Brandt E, et al. The testing process in Family Medicine: Problems, Solutions and Barriers as Seen by Physicians and their Staffs. A study of the American Academy of Family Physicians' National Research Network. J Patient Safety 2006;2:25-32.

7 Elder N, McEwen T, Flach J, et al. Creating safety in the testing process in primary care offices. Rockville, MD: Agency for Healthcare Research and Quality, 2008. http://www.ahrq.gov/ downloads/pub/advances2/vol2/Advances-Elder_18.pdf

8 Elder N, McEwen T, Flach J, et al. The management of test results in family medicine offices. Ann Fam Med 2009;7:343-51.

9 Elder NC, McEwen TR, Flach J, et al. The management of test results in primary care: does an electronic medical record make a difference? Fam Med 2010;42:327-33.

10 Graham DG, Harris DM, Elder NC, et al. Mitigation of patient harm from testing errors in family medicine offices: a report from the American Academy of Family Physicians
National Research Network. Qual Saf Health Care 2008;17:201-8.

11 Hickner J, Graham D, Elder N, et al. Testing process errors and their harms and consequences reported from family medicine practices: A study of the AAFP National Research Network. Qual Saf Health Care 2008;17:194-200.

12 Hickner JM, Fernald DH, Harris DM, et al. Issues and initiatives in the testing process in primary care physician offices. Jt Comm J Qual Patient Saf 2005;31:81-9.

13 Poon EG, Gandhi TK, Sequist TD, et al. "I wish I had seen this test result earlier!": dissatisfaction with test result management systems in primary care. Arch Intern Med 2004;164:2223-8.

14 Poon EG, Wang SJ, Gandhi TK, et al. Design and implementation of a comprehensive outpatient Results Manager. J Biomed Inform 2003;36:80-91.

15 Woolf SH, Kuzel AJ, Dovey SM, et al. A String of mistakes: the importance of cascade analysis in describing, counting, counting and preventing medical errors. Ann Fam Med 2004;2:317-26.

16 Litchfield IJ, Bentham LM, Lilford RJ, et al. Patient perspectives on test result communication in primary care: a qualitative study. Br J Gen Pract 2015;65:e133-40.

17 Litchfield IJ, Bentham LM, Lilford RJ, et al. Test result communication in primary care: clinical and office staff perspectives. Fam Pract 2014;31:592-7.

18 Litchfield I, Bentham L, Hill A, et al. Routine failures in the process for blood testing and the communication of results to patients in primary care: a qualitative exploration of patient and provider perspectives. BMJ Qual Saf 2015;24: 681-90.

19 Litchfield I, Bentham L, Lilford R, et al. Test result communication in primary care-a survey of current practice. BMJ Qual Saf 2015;24:691-9.

20 Elder NC, Miller WL. Reading and evaluating qualitative research studies. J Fam Pract 1995;41:279-85.

21 Cohen DJ, Crabtree BF. Evaluative criteria for qualitative research in health care: controversies and recommendations. Ann Fam Med 2008;6:331-9.

22 Moon MD, Wolf LA, Baker K, et al. Evaluating qualitative research studies for use in the clinical setting. J Emerg Nurs 2013;39:508-10.

23 Nutting PA, Miller WL, Crabtree BF, et al. Initial lessons from the first national demonstration project on practice transformation to a patient-centered medical home. Ann Fam Med 2009; 7:254-60.

24 Bowie P, Halley L, McKay J. Laboratory test ordering and results management systems: a qualitative study of safety risks identified by administrators in general practice. BMJ Open 2014;4:e004245.

25 Beasley JW, Wetterneck TB, Temte J, et al. Information chaos in primary care: implications for physician performance and patient safety. J Am Board Fam Med 2011;24:745-51.

26 Smith PC, Araya-Guerra R, Bublitz C, et al. Missing clinical information during primary care visits. JAMA 2005;293:565-71.

27 Kern LM, Callahan MA, Brillon DJ, et al. Glucose testing and insufficient follow-up of abnormal results: a cohort study. BMC Health Serv Res 2006;6:87.

28 Elder NC, Pallerla H, Regan S. What do family physicians consider an error? A comparison of definitions and physician perception. BMC Fam Pract 2006;7:73. 


\section{Editorial}

29 Baldwin DM, Quintela J, Duclos C, et al. Patient preferences for notification of normal laboratory test results: a report from the ASIPS Collaborative. BMC Fam Pract 2005;6:11.

30 Elder NC, Barney K. "But what does it mean for me?" Primary care patients' communication preferences for test results notification. Jt Comm J Qual Patient Saf 2012;38:168-76.

31 Giardina T, Modi V, Parrish D, et al. The patient portal and abnormal test results: an exploratory study of patient experiences. Patient Experience Journal 2015;2:148-54.

32 Grayston J, Fairhurst K, McKinstry B. Using new technologies to deliver test results in primary care: structured interview study of patients' views. Prim Health Care Res Dev 2010;11:142-54.

33 Leekha S, Thomas KG, Chaudhry R, et al. Patient preferences for and satisfaction with methods of communicating test results in a primary care practice. Jt Comm J Qual Patient Saf 2009;35:497-501.

34 Petrie KJ, Muller JT, Schirmbeck F, et al. Effect of providing information about normal test results on patients' reassurance: randomised controlled trial. BMJ 2007;334:352.

35 Elder NC, Jacobson C, Zink T, et al. How Experiencing Preventable Medical Problems Changed Patients' Interactions With Primary Health Care. Ann Fam Med 2005;3:537-44. 